\title{
Identificación y Localización de Fallas en Sistemas de Distribución con Medidores de Calidad del Servicio de Energía Eléctrica
}

\author{
Víctor A. Gómez ${ }^{(1)}$, Robín A. Peña ${ }^{(2)}$, Cesar Hernández ${ }^{(3)^{*}}$
}

(1) Universidad Distrital Francisco José de Caldas, investigador del grupo ARMOS, Bogotá-Colombia (e-mail: victorgomez8807@gmail.com)

(2) Universidad Distrital Francisco José de Caldas, investigador del grupo ARMOS, Bogotá-Colombia (e-mail: alejandro2905@gmail.com)

(3) Universidad Distrital Francisco José de Caldas, Docente e investigador del grupo ARMOS, Bogotá-Colombia (e-mail: cahernandezs@udistrital.edu.co)

*Autor a quien debe ser dirigida la correspondencia

Recibido Sep. 01, 2011; Aceptado Nov. 03, 2011; Versión Final recibida Nov. 10, 2011

\section{Resumen}

Se propone una nueva metodología para la identificación y localización de fallas en sistemas de distribución de energía eléctrica, a través de la instalación de medidores electrónicos para registrar la continuidad en el servicio eléctrico. Estos medidores, facilitan la detección de fallas permanentes o transitorias en redes no homogéneas, proporcionando información sobre puntos débiles de los sistemas de potencia, permitiendo adoptar medidas correctivas con respecto a la duración y frecuencia de las interrupciones, en los circuitos de los sistemas de distribución local. La propuesta planteada mitiga la discontinuidad del servicio y los cuantiosos costos que producen a los agentes de sector eléctrico y principalmente a los usuarios de la energía eléctrica.

\section{Identification and Location of Distribution Systems Failures Through Service Quality Meters of Electric Power}

\begin{abstract}
A new methodology for identifying and locating faults in distribution systems of electric power is proposed. The installation of electronic meters to record the electrical service continuity is proposed. These meters facilitated the detection of permanent faults or transient nonhomogeneous networks, providing information about weaknesses in power systems. This allows adopting corrective action with respect to the duration and frequency of interruptions in the circuits of local distribution systems. The proposal mitigates the discontinuity of the service and the significant costs for the electricity companies and especially for the users of electricity.
\end{abstract}

Keywords: electricity interruptions, electrical continuity, distribution systems, faults 


\section{INTRODUCCIÓN}

A partir de la expansión de los sistemas de distribución de energía eléctrica en el mundo, el problema de la discontinuidad en el servicio, se ha convertido en un tema de gran interés para los agentes del sector eléctrico, en las áreas de planeación, operación, distribución, y más aún, para los usuarios residenciales e industriales. Tanto las empresas prestadoras del servicio de energía eléctrica, como lo usuarios, interactúan con respecto a la valoración de los costos debidos a las interrupciones del suministro de energía eléctrica (Alfonso et al., 2005). Con los procesos de globalización, las empresas prestadoras del servicio de electricidad del mundo, realizan estudios para valorar los costos debidos a una inadecuada continuidad en el suministro, y de esta forma, obtener un punto equidistante entre el costo de inversión para mejorar la confiabilidad de un sistema y los costos que las interrupciones representan a los usuarios del servicio (Sullivan et al., 1995; Sullivan et al., 1996).

La localización de fallas no es un problema nuevo. Sin embargo, las investigaciones que se han realizado en esta área recaen sobre los sistemas de transmisión donde las características homogéneas de la línea, la medición en ambos terminales y la disponibilidad de más equipos, permiten localizar el sitio de falla con alta precisión. La localización en sistemas de distribución es más problemática, por la complejidad debida a la presencia de conductores no homogéneos, cargas intermedias, laterales y desbalance del sistema y de la carga (Morales et al., 2009). Con lo cual se evidencia, que un sistema de distribución local es más vulnerable a los diferentes factores que afecten la continuidad del servicio prestado, por la singularidad de las topologías de sus redes eléctricas. A partir de la investigación desarrollada, se busca presentar el método de implementación de medidores electrónicos, que permiten tener un mejor esquema de regulación y registro de los sistemas de potencia, para mitigar la incidencia de factores como la discontinuidad en el servicio eléctrico (Carvajal, 2009).

Los estudios sobre continuidad o confiabilidad en los sistemas eléctricos han estado enfocados en las áreas de generación y cogeneración en el que se destaca la homogeneidad de los sistemas de potencia. Sin embargo, desde 1960 han existido algunos pocos estudios para la determinación de la confiabilidad en transmisión y distribución, y en los puntos de carga (Baeza et al., 2003). Tales estudios han representado, el diseño de metodologías a partir de algoritmos de identificación de fallas, basados en el historial de eventos ocurridos y en lo establecido por los entes de regulación (Anaut, 2009; Gómez, 2011).

Desde mediados del siglo XX hasta nuestros días, el sector eléctrico del mundo ha crecido de manera exponencial. Por ejemplo: las empresas distribuidoras de Estados Unidos experimentaron el comienzo de un rápido aumento en el número de usuarios de energía eléctrica. Este crecimiento de la demanda, provocó la expansión de los sistemas de planeación y distribución. A medida que los sistemas de distribución crecieron en complejidad, la continuidad del servicio se volvió un tema de gran importancia para los ingenieros eléctricos, y más aún, cuando el 14 de agosto del 2003, los países: Estados Unidos y Canadá, experimentaron el mayor apagón de la historia, afectando a cerca de 50 millones de personas por la ausencia de electricidad con una duración aproxima de 29 horas. Se estima, que las pérdidas fueron más de \$USD 5.000 millones.

Se calcula que el costo de los apagones que actualmente sufre Estados Unidos llega a los \$USD 120.000 millones; el plan energético que planteó el presidente George W. Bush buscaba ahorrar energía mediante el uso adecuado de la tecnología, además de presentar recomendaciones para modernizar el sistema eléctrico de los Estados Unidos (Torres et al., 2003). Con todas las experiencias vividas en el mundo con respecto a deficiencias en la calidad de la energía eléctrica; organizaciones internacionales como la International Electrotechnical Commission (IEC) y el Institute of Electrical and Electronics Engineers (IEEE) desarrollaron una serie de normas y métodos de valoración directa de costos debidos a interrupciones (Dialynas et al., 2001; Sullivan et al., 1995; Sousa y Martins, 2003).

Mejorar la calidad del servicio prestado es de vital importancia para una sociedad actual en donde la energía eléctrica es indispensable en la mayoría de las actividades del ser humano, tanto en 
labores cotidianas domésticas e industriales. Con el avance de las tecnologías, la mayoría de equipos eléctricos y electrónicos de uso doméstico e industrial, son más vulnerables a variaciones de los parámetros de alimentación. Por ende, la calidad de la energía y la continuidad en el servicio de suministro, deben regirse bajos principios de confiabilidad. En consecuencia es necesario adoptar metodologías que faciliten la rápida y confiable identificación de fallas y de esta manera establecer medidas que mitiguen el impacto económico y social, asociado los factores característicos de la energía eléctrica. Las inversiones que hacen los operadores de red y los comercializadores para mejorar dicha calidad del servicio, se debe evidenciar de manera clara y transparente en interrupciones menos frecuentes y prolongadas, plasmadas en datos de indicadores veraces y definitivos.

\section{IDENTIFICACIÓN Y LOCALIZACIÓN DE FALLAS}

El diagnostico de fallas en las redes de distribución es de gran importancia incluso en las redes de media tensión. Del mismo modo, la identificación y localización de tales eventos es un importante factor para la calidad del servicio. Para el diagnostico de fallas se emplean algoritmos que consisten principalmente en el cómputo de la impedancia de la línea de falla, basados en el voltaje y la corriente registrados directamente en el alimentador primario. Por lo tanto, se establece una relación entre la impedancia y la distancia de la falla, teniendo presente los parámetros de la línea (Sullivan et al., 1996). Este método es eficiente para los sistemas de transmisión porque permite detectar en un punto preciso la ocurrencia de la interrupción. Sin embargo, tal algoritmo no es aplicable a redes de distribución, debido a que estas, presentan topologías mucho más complejas, en la cual, la distancia asociada con la impedancia estimada, puede coincidir con diferentes puntos de un sistema ramificado.

\section{Índices en Colombia}

Actualmente las empresas suministradoras del servicio de energía, tienen un valor máximo admisible de interrupciones que pueden utilizar a lo largo del año, para trabajos de mantenimiento y expansión de las redes de distribución. Para el caso de Latinoamérica, Colombia, estableció para el año 2010, los índices máximos admisibles en la duración y frecuencia de la interrupciones. En la tabla No. 1 se describen los índices para cada trimestre del año. Si la empresa prestadora del servicio supera los valores máximos admisibles, deberá indemnizar a los usuarios por la falta en la prestación del servicio público.

Tabla 1: Indicadores de calidad 2010 (valores máximos admisibles). DES: Tiempo de interrupción del servicio en un circuito (horas); FES: Número de interrupciones del servicio en un circuito [E.P.M. 2010]

\begin{tabular}{|c|c|c|c|c|c|c|c|c|c|c|}
\hline \multirow{2}{*}{ Grupo } & \multicolumn{2}{|c|}{ Índices Anuales } & \multicolumn{2}{c|}{ Ene, Feb, Mar } & \multicolumn{2}{c|}{ Abr, May, Jun } & \multicolumn{2}{c|}{ Jul, Ago, Sep } & \multicolumn{2}{|c|}{ Oct Nov, Dic } \\
\cline { 2 - 11 } & DES & FES & DES & FES & DES & FES & DES & FES & DES & FES \\
\hline 1 & 11 & 23 & 2.7 & 7 & 3.2 & 7 & 2.9 & 6 & 2.2 & 6 \\
\hline 2 & 19 & 44 & 5.0 & 12 & 6.0 & 12 & 4.5 & 10 & 3.5 & 10 \\
\hline 3 & 29 & 51 & 7 & 10 & 9 & 16 & 7 & 15 & 6 & 10 \\
\hline 4 & 39 & 58 & 9 & 13 & 12 & 17 & 11 & 16 & 7 & 12 \\
\hline
\end{tabular}

\section{Esquema de monitorización}

En la Fig. 1 se logra observar, un diagrama del sistema de distribución, en el cual la subestación principal o alimentador primario (1) está encargada de suplir energía eléctrica a usuarios residenciales ( 2 y 3 ) e industriales (8) a través de líneas de distribución $(4,5$ y 6 ) y de un nodo de 
interconexión (7). Los operadores de red miden la continuidad del servicio de energía directamente sobre la subestación (1). Entonces, si ocurre una falla en los puntos (4, 5, 6 ó 7), esta interrupción no será oportunamente registrada por los medidores de la subestación, debido a que estos solo monitorizan los interruptores que se encuentran alojados en la misma. En consecuencia, los usuarios residenciales e industriales serán los principales afectados por la discontinuidad del servicio, que repercutirá en altas perdidas en productividad, daño en equipos, productos y procesos. Todo lo anterior, por la tardanza en la identificación y localización de la falla eléctrica, para su posterior re-conexión. Se estima que anualmente Estados Unidos tiene pérdidas superiores a los 25 billones de dólares, por las interrupciones en el servicio de energía, por fenómenos naturales u ocasionados por una mala coordinación o supervisión de los sistemas de transmisión y distribución (Sousa y Martins, 2003).

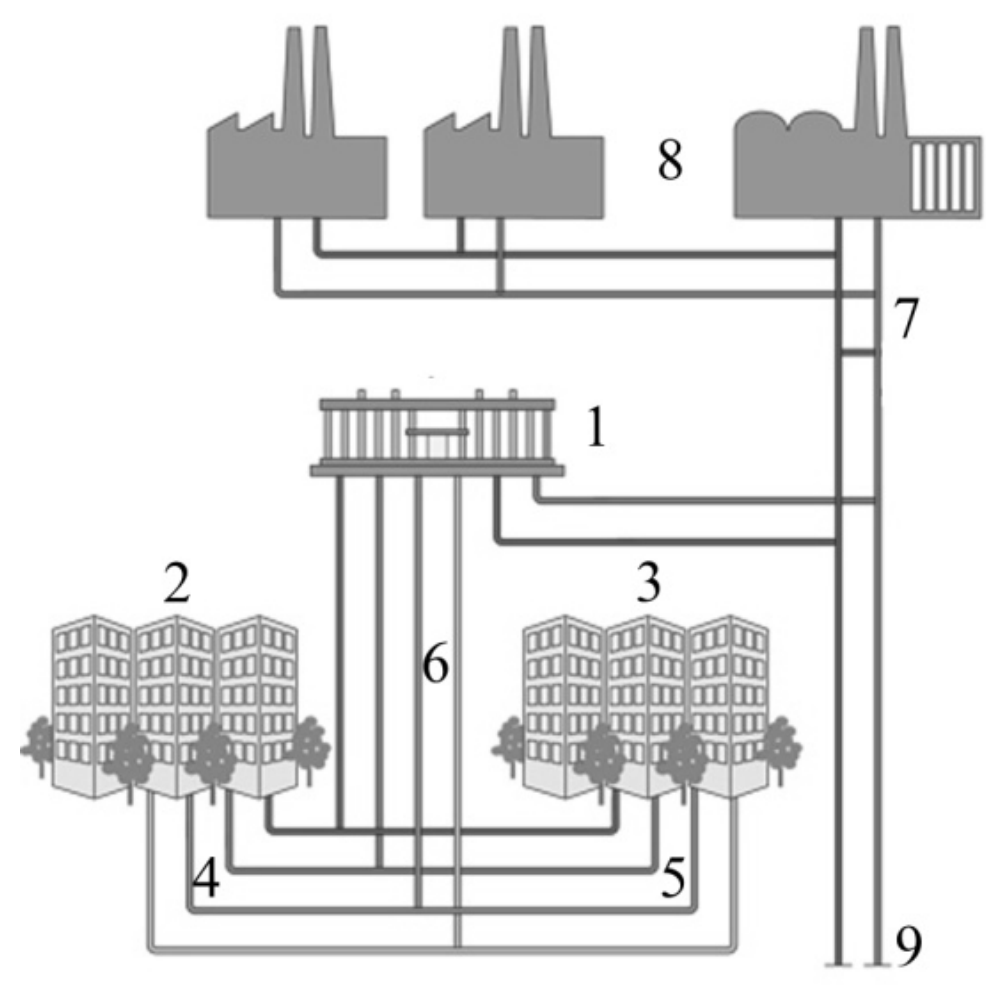

Fig. 1: Diagrama del sistema de distribución. 1: Subestación principal en la red de distribución; 2 y 3: Usuarios residenciales; 4, 5 y 6: Red eléctrica de distribución; 7: Nodo de conexión usuarios Industriales; 8: Usuarios industriales; 9: Red principal de distribución

\section{METODOLOGÍA}

Frente a la problemática planteada en relación a la identificación y localización de fallas en sistemas de distribución, el grupo de investigación de arquitecturas modernas para sistemas de alimentación (ARMOS) de la Universidad Distrital Francisco José de Caldas, desarrolló un primer prototipo electrónico con el nombre de: Medidor de indicadores de calidad en el servicio de energía eléctrica (DES y FES) para usuario residencial (Gómez et al., 2011); concebido inicialmente como un registrador enfocado a la regulación de los sistemas DES de los usuarios suscriptores del servicio energético. La funcionalidad del medidor se ha ampliado de forma progresiva, mediante la incorporación de nuevos esquemas de medición, algoritmos de control y sistemas de comunicación. Para orientar de manera más general, a la solución de problemas de electrotecnia, enfocados a la calidad de la potencia eléctrica y la calidad del servicio eléctrico Zapata, 2011). 


\section{Medidores electrónicos}

La gran mayoría de los sistemas de distribución poseen topologías de tipo radial. Por tanto, es necesario disponer de medidores electrónicos en diferentes puntos del sistema de conexión. Los nodos de análisis pueden ser: transformadores de media y baja tensión, interruptores de potencia, barrajes, entre otros. Entre más puntos se tomen del sistema eléctrico para la identificación y localización de fallas, mayor será la precisión de diagnóstico de discontinuidad en el servicio energético. Al igual, los medidores electrónicos, pueden ser implementados directamente en usuarios residenciales e industriales. De ser este el caso, los medidores deben ser instalados directamente en el armario que contiene los registradores de energía activa, específicamente en el barraje de alimentación de los diferentes circuitos. En la Fig. 2 y 3 se observa la instalación.

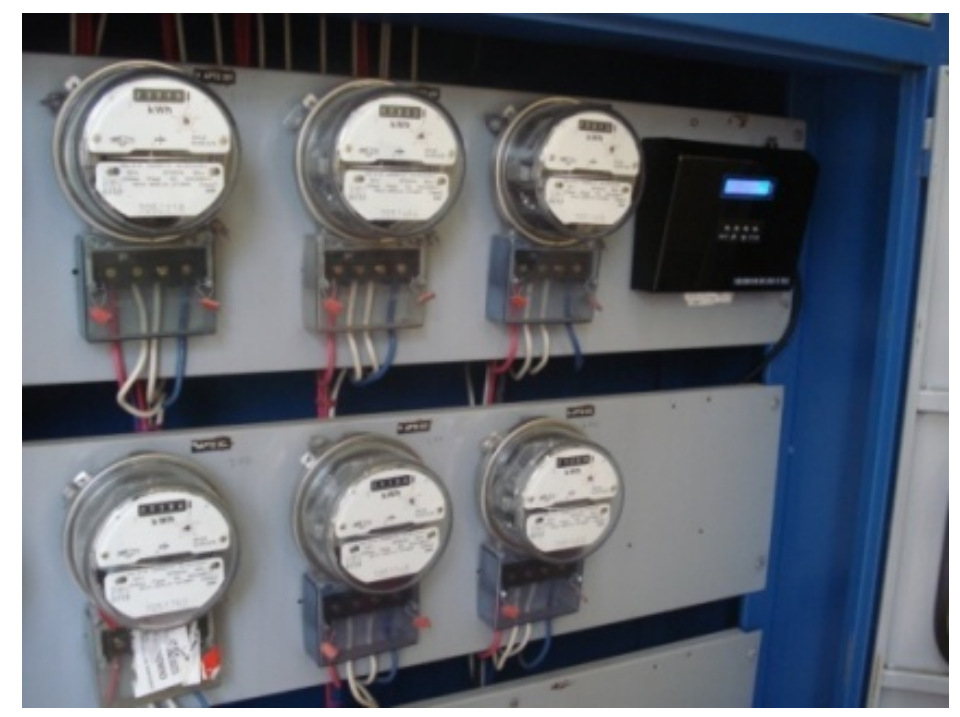

Fig. 2: armario de medidores

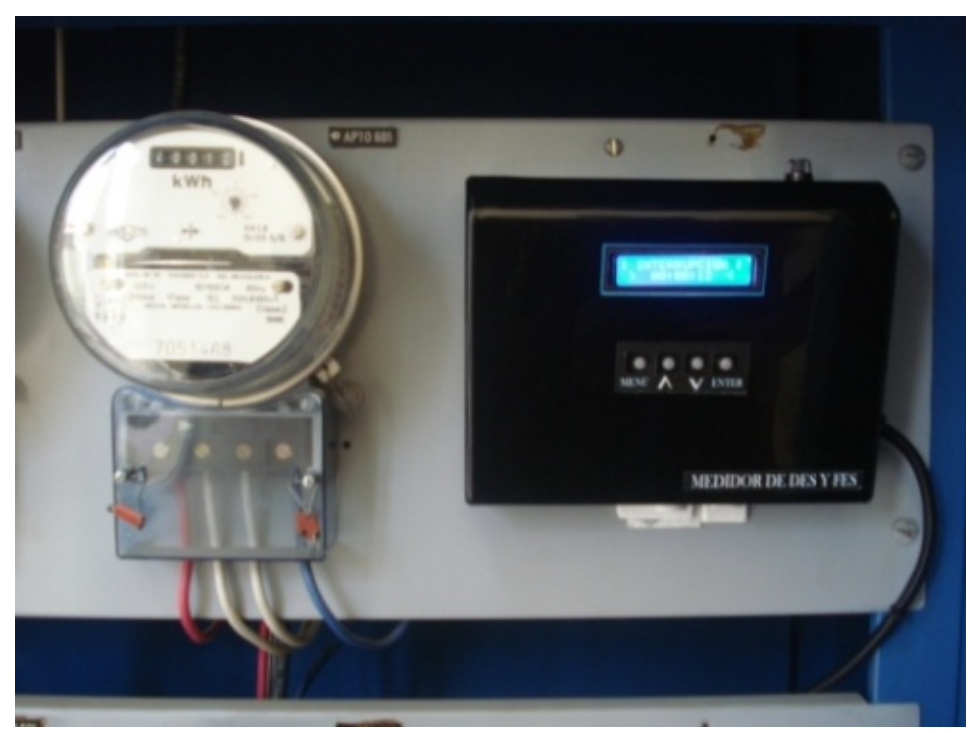

Fig. 3: medidor de energía activa y medidor de continuidad en el servicio de energía eléctrica

El acondicionamiento del sensor de voltaje que se encuentra alojado en el medidor electrónico, depende del nivel de tensión del nodo de interconexión de la red de distribución local, donde será instalado el prototipo; por lo que, se pueden encontrar diferentes niveles de tensión. La instalación de los medidores debe realizarse por personal calificado, cumpliendo las normas nacionales e internacionales. Para el caso Colombiano la instalación se debe regir bajos los principios establecidos en la norma técnica Colombiana 2050 (NTC 2050) y el Reglamento técnico de instalaciones eléctricas (RETIE). 


\section{Detección de eventos de tensión}

Se debe censar la componente fundamental de la señal de voltaje al momento de pre-falla y falla, debido a que pueden existir interrupciones permanentes o transitorias. El colapso de una falla presente en el sistema de distribución local, ocasiona cuantiosas pérdidas económicas a los pequeños y grandes consumidores. Por tal razón, el medidor electrónico censa la ausencia de tensión e informa a los especialistas para que se genere un rápido aislamiento, reparación y restauración del servicio energético. Con lo anterior, se mitigarían los altos índices de la duración de las interrupciones presentes en un circuito (DES). Las interrupciones transitorias no se pueden identificar por simple inspección, por tal razón, el medidor electrónico, permite detectar los puntos débiles de los sistemas de potencia, en donde reincide la falla. De esta manera, se logra tener un esquema de regulación, para atenuar los altos índices del indicador de frecuencia equivalente en las interrupciones de un circuito (FES).

\section{Sistema de comunicación}

A partir de la necesidad de obtener los registros de los medidores en los diferentes puntos de la red de distribución, se debe implementar un sistema de transmisión inalámbrico, adecuado para dar soporte a las necesidades de telemetría. Tal comunicación, es establecida mediante estándares ZigBee, por su bajo costo, soporte para múltiples topologías de red, rango de alcance y bajo consumo de energía.

Este sistema de comunicación, permite obtener un estudio en tiempo real de la información adquirida por el medidor electrónico, frente a un suceso característico en las líneas de distribución. Por tanto, el medidor emite un diagnostico que informa a los especialistas (principalmente a los operadores de las subestaciones) de los eventos de ocurrencia en las redes de distribución local.

Una vez emitido un diagnostico por el medidor; el receptor de la información ubicado en la subestación principal, migrará los datos recibidos hacia el ordenador de la subestación, a través del software que se observa en la Fig. 4. Seguidamente, este programa generará un reporte de la ocurrencia de los eventos con fecha, hora y emitirá la identificación de la línea que ha salido de servicio. Con base en este informe, el operador o el especialista de turno podrá, emitir un diagnostico a seguir para proceder a recuperar el sistema de distribución local. Es decir, restablecer el servicio de energía para los usuarios.

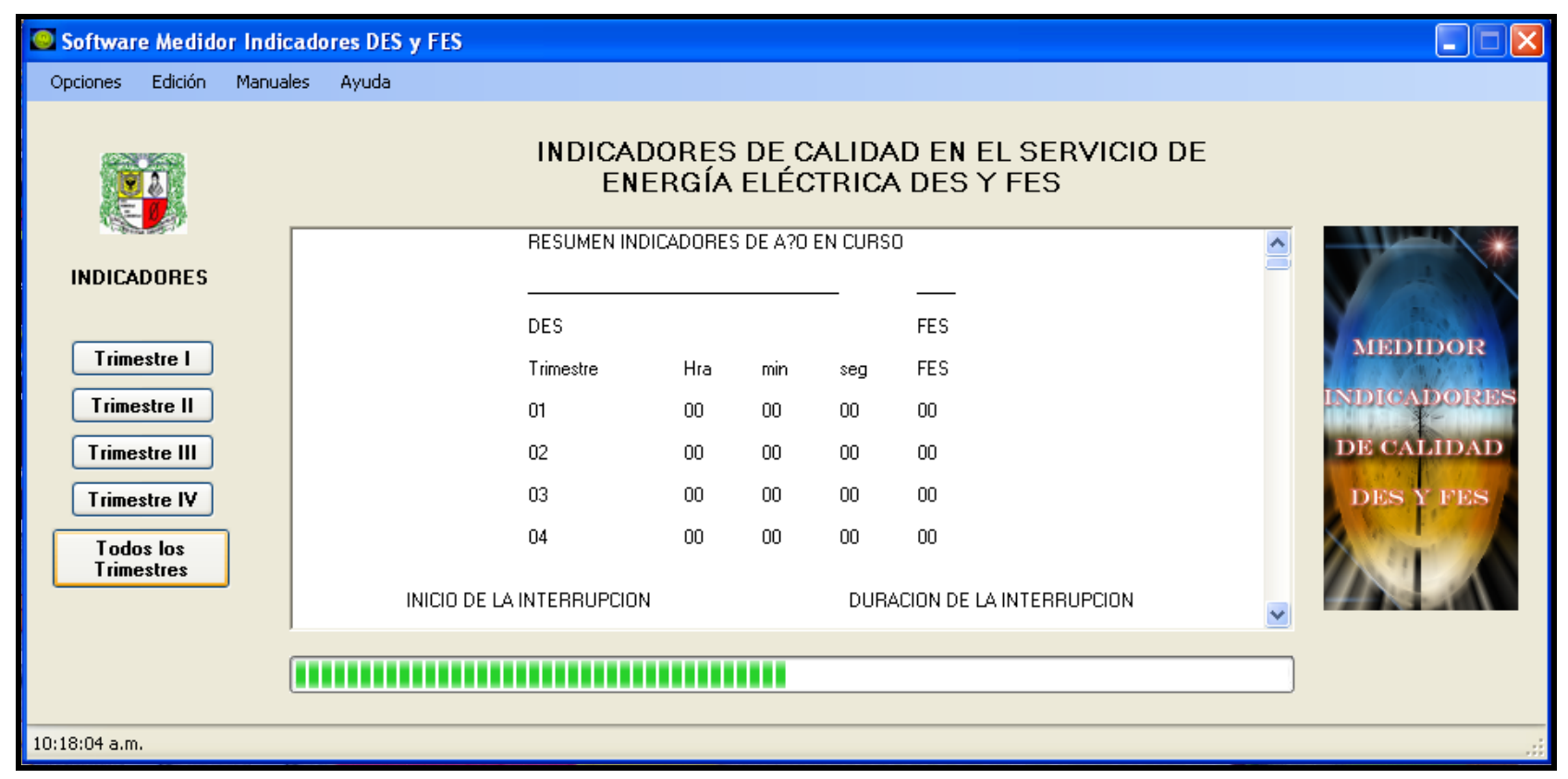

Fig. 4: Software "DES y FES" 
Dentro de la regulación Colombiana, está establecido que los operadores de red y los comercializadores de la energía eléctrica deben generar reportes de los indicadores DES y FES de manera trimestral, para ser presentados a organismos de control y regulación. En relación a lo anterior, el software cuenta con la posibilidad de observar el historial de ocurrencia de eventos con información de la duración, fecha y localización de la falla en el sistema de los últimos 12 meses. Por tanto, el programa, permite tener una mejor inspección de las características de las interrupciones en los circuitos.

\section{CONCLUSIONES}

El nuevo esquema de regulación de la energía eléctrica y la competencia de los mercados nacionales e internacionales, ha hecho que las empresas prestadoras del servicio de energía eléctrica, adopten nuevas metodologías para el mejoramiento del servicio prestado hacia usuarios de sistemas de distribución industrial, comercial, urbano y rural.

La implementación de medidores que registren la continuidad del servicio de energía, permite tener un mejor control e inspección de los sistemas de distribución. Al igual, facilita la oportuna y rápida localización de fallas, mitigando los elevados costos de compensaciones, hechas por los diferentes agentes del sector eléctrico, debidos a una inadecuada continuidad en el suministro de energía. Todo lo anterior, a fin de remediar la ausencia del servicio energético en los usuarios finales.

La adopción de nuevas metodologías para la identificación de fallas en sistemas de distribución, partiendo de sistemas expertos de monitorización, permite aminorar los indicadores de duración y frecuencia de las interrupciones del servicio eléctrico.

El correcto diagnóstico de fallas en las redes eléctricas, permite evitar colapsos en los sistemas de transmisión y distribución de electricidad. Además, permite que los usuarios y los agentes del sector eléctrico sean protagonistas en el mejoramiento del sector energético del mundo.

\section{AGRADECIMIENTOS}

Este trabajo de investigación se desarrolló gracias al apoyo del Centro de Investigaciones y Desarrollo Científico de la Universidad Distrital Francisco José de Caldas.

\section{REFERENCIAS}

Anaut, D., Di Mauro, M.G. y Suarez, J., Optimización de Redes Eléctricas Mediante la Aplicación de Algoritmos Genéticos, Información Tecnológica: 20 (4), 137-148 (2009).

Alfonso, P., Jenny, P., Jairo H. y Gabriel O. y J. Benjamín. Methodological proposal for cost valuation due to an inadequate continuity in the electric energy supply. III International Symposium on Electric Energy Quality SICEL 2005, Bogotá 16 a 18 November (2006).

Baeza, R., J. Rodríguez y J. Hernández, evaluación de confiabilidad de sistema de distribución eléctrica en desregulación. Revista Facultad de ingeniería, U.T.A. (Chile): 1(1), 33-39 (2003).

Carvajal, S., López J. y Lemoine C., Reconfiguración de sistemas de distribución de energía eléctrica usando un algoritmo de optimización basado en colonia de hormigas, Tecnura: 12 (24), 14-22 (2009).

Dialynas E. N., S. M. Megaloconomus y Dali V. C. Interruption Cost Analysis for the Electrical Power Customers in Greece. 6th International Conference and Exhibition on Electricity Distribution (CIRED 2001), 129-129 Amsterdam 18 a 21 Junio (2001). 
Gómez G., Carreño J. y Caviedes J., Herramienta de software para el pronóstico de demanda horaria de potencia eléctrica en el sistema eléctrico de Codensa S.A. Esp., Tecnura: 15 (28), 7-22 (2011).

Gómez V., Peña R. y Hernández C., Diseño y construcción de un medidor de indicadores de calidad en el servicio de energía eléctrica (DES y FES) para usuario residencial. Ingeniería y desarrollo: 7(2), 101-112 (2011).

Morales G., R. Barrera, y Vargas H., Ubicación única de fallas en sistemas de distribución por medio de zonas con SVM. Revista Facultad de Ingeniería Universidad de Antioquia, ISSN: 01200230, 47, 187-196 (2009).

REPORT: Empresas Publicas de Medellin. In compliance with Act 142 of 1994 and the CREG resolutions 058 and 119 of 2000 and 078 of 2007. Maximum allowable values (2010).

Sullivan M., T. Vardell y M. Johnson, Power Interruption Costs to Industrial and Commercial Consumers of electricity. Industrial and Commercial Power Systems Technical Conference, 1996: 23-35, USA 6 a 9 Mayo (1996).

Sullivan M., T. Vardell, N. Suddeth y A. Vojdani, Interruption Costs, Customer Satisfaction and Expectations for Service Reliability. IEEE Transactions on Power Systems: 11(2), 989-995 (1995).

Sousa Martins L., J. F. Martins, Member IEEE, C. M. Alegria and V. Fernao Pires, Member IEEE, A network distribution power System Fault location based on neuronal eigenvalue algorithm. 2003 Torres H., G. Acero, J. Flechas, J. Saucedo y C. Quintana, Electric Power, a quality product, ICONTEC. ISBN 958-9383-38-6, Bogotá (2003).

Zapata G. y Chacón E., Aportes Metodológicos para el Diseño de Sistemas de Supervisión de Procesos Continuos, Información Tecnológica: 22 (3), 97-114 (2011). 